\title{
A narrative review of non-pharmacological management of SARS-CoV-2 respiratory failure: a call for an evidence based approach
}

\author{
Rishik Vashisht ${ }^{1}$, Sudhir Krishnan ${ }^{1,2}$, Abhijit Duggal ${ }^{1,2}$ \\ ${ }^{1}$ Department of Critical Care Medicine, Respiratory Institute, Cleveland Clinic Foundation, Cleveland, USA; ${ }^{2}$ Department of Medicine, Cleveland \\ Clinic Lerner College of Medicine of Case Western Reserve University, Cleveland, USA \\ Contributions: (I) Conception and design: R Vashisht, A Duggal; (II) Administrative support: S Krishnan; (III) Provision of study materials or patients: \\ None; (IV) Collection and assembly of data: None; (V) Data analysis and interpretation: None; (VI) Manuscript writing: All authors; (VII) Final \\ approval of manuscript: All authors. \\ Correspondence to: Abhijit Duggal, MD, MPH, MSc. Department of Critical Care Medicine, Respiratory Institute, 9500 Euclid Avenue, Cleveland, \\ Ohio 44195, USA. Email: Duggala2@ccf.org.
}

\begin{abstract}
A novel severe acute respiratory syndrome coronavirus (SARS-CoV-2) belonging to genus beta-coronavirus has been associated with an acute respiratory disease termed coronavirus disease 2019 (COVID-19). As of September 3, 2020, SARS-CoV-2 had caused 867,219 fatalities in 188 nations across the globe. Rapid progression to bronchopneumonia manifesting with severe hypoxemia and eventual evolution into acute respiratory distress syndrome (ARDS) necessitating mechanical ventilation is the hallmark of this disease. The novel nature of COVID-19 pneumonia and the high morbidity and mortality associated with the same has vexed the critical care community. A cultural shift away from evidence-based medicine, and the impetus to attempt newer unproven therapies like awake proning, interleukin receptor 6 antagonists, inhaled nitric oxide, empiric anticoagulation etc. over modalities that have been tested over the decades is slowly gaining ground. The suggestions to delay intubations and liberalize tidal volumes have polarized the medical field like never before. The lack of consistency in management practices and establishing practices based on anecdotes and experiences can lead to devastating outcomes in the patients affected by this deadly virus. In this narrative review, we attempt to re-emphasize the need for an evidence-based approach to the management of COVID-19 related ARDS and review treatment strategies that have been established after rigorous trials and have stood the test of time.
\end{abstract}

Keywords: Acute respiratory distress syndrome (ARDS); coronavirus disease 2019 (COVID-19); mechanical ventilation; severe acute respiratory syndrome coronavirus (SARS-CoV-2)

Submitted Jun 11, 2020. Accepted for publication Sep 18, 2020.

doi: $10.21037 /$ atm-20-4633

View this article at: http://dx.doi.org/10.21037/atm-20-4633

\section{Introduction}

A novel severe acute respiratory syndrome coronavirus (SARS-CoV-2) belonging to genus beta-coronavirus has been associated with an acute respiratory disease termed coronavirus disease 2019 (COVID - 19) by the International Committee on Taxonomy of Viruses (1). The World Health Organization (WHO) declared the SARSCoV-2 outbreak a pandemic on March 11, 2020 (2). As of
September 3, 2020, SARS-CoV-2 had infected 26,208,690 people in 188 countries/regions with 867,219 deaths (3). Depending upon the country, the case fatality rate (CFR) ranges from $0.25 \%$ to $10 \%$ (4). However, due to the lack of standardized criteria for testing and for the recording of deaths, the real mortality rate and CFR will be unknown and will likely change once the actual prevalence of the disease becomes apparent. COVID-19 is associated with 
a $5 \%$ to $14 \%$ intensive care unit (ICU) admission rate $(5,6)$. Between $30-88 \%$ of critically ill patients require mechanical ventilation (7). High mortality of up to $88 \%$ has been reported with need for mechanical ventilation in these patients (6). These initial reports have significant flaws in the way they are describing their outcomes and likely suffer from both a selection and reporting bias, as has been described in previous pandemics (8). Unfortunately such selective reporting has led to a to a negative perception that outcomes associated with mechanical ventilation are much worse in these patients when compared to other causes of respiratory failure and acute respiratory distress syndrome (ARDS), both among the public and the lay media (9).

COVID-19 respiratory illness commonly presents with fever, fatigue, dry cough, myalgias and dyspnea. SARSCoV-2 leads to diffuse alveolar damage (predominant involvement of type II alveolar cells) with fibrin rich hyaline membranes and a few multinucleated giant cells (10). The renin-angiotensin system (RAS) arm disequilibrium caused by SARS-CoV-2 has been hypothesized to be the pathophysiological hallmark of the disease. Binding of the SARS-CoV-2 to angiotensin-converting enzyme 2 (ACE2) causes its functional downregulation, thereby enhancing the classic renin angiotensin system (RAS) and attenuating the anti-RAS arm. This disequilibrium leads to an intense inflammatory response resulting in leaky pulmonary capillaries and eventual fibrosis (11). Rapid progression to bronchopneumonia manifesting with severe hypoxemia and eventual evolution into ARDS necessitating mechanical ventilation is the clinical hallmark of this medical scourge (12).

The novel nature of COVID-19 pneumonia and the high morbidity and mortality associated with the same has vexed the critical care community. The disease has overwhelmed hospital systems in major parts of the world (13). A sense of desperation appears to pervade the care of the critically ill COVID-19 patients. Anxiety to turn the tide of the rising death toll, somehow and anyhow, seems palpable and appears to govern at least some of the therapeutic decisions undertaken by the medical community. A cultural shift away from evidence based medicine, and the impetus to attempt newer unproven therapies over modalities that have been tested over the decades (14) is slowly gaining ground. Casting aside lessons learned over two decades of ARDS management in favor of anecdotal therapies (interleukin receptor 6 antagonists, inhaled nitric oxide, empiric anticoagulation etc.) or management strategies (liberalization of tidal volume, low positive end expiratory pressure or PEEP, delayed intubation etc.), however well intentioned, does not augur well for our patients or the practice of critical care. In this review, we attempt to reemphasize the need for an evidence-based approach to the management of COVID-19 related ARDS and review treatment strategies that have stood the test of time (Figure 1). We present the following article in accordance with the Narrative Review reporting checklist (available at http://dx.doi.org/10.21037/atm-20-4633).

\section{Methods}

We conducted a literature search for articles published in English language up to July, 2020, on the respiratory failure in patients Infected With SARS-CoV-2 and standard ARDS management, using three medical databases: PubMed, Google Scholar, and Web of Science. We used the following keywords (including specific Medical Subject Headings, MeSH): "COVID-19/respiratory failure", "COVID-19/ ARDS", "ARDS/management" and "ARDS/mechanical ventilation. We used other free text terms like: "COVID-19 phenotypes", "awake proning", "high flow nasal cannula" and "HEPA filters". The relevant articles were reviewed and selected by all the authors. We reviewed the bibliography of the selected articles to identify studies that might have been missed during the initial search.

\section{Discussion}

\section{Management of acute respiratory failure}

\section{Oxygen therapy}

Patients with COVID-19 pneumonia have rapidly escalating oxygen requirements, and should receive supplemental oxygen through a high flow nasal cannula (HFNC) to maintain oxygen saturation between 92-96\% (15). If the resources are available these patients should be monitored in an ICU. In resource poor settings, or in surge situations institute specific contingency planning needs to be in place for the appropriate care of these patients (16).

In acute hypoxemic respiratory failure (AHRF), HFNC has been shown to decrease intubation rates when compared to conventional oxygen in patients [9 RCTs; n=2,093; RR 0.85, 95\% confidence interval (CI), 0.74-0.99] (17). Non-invasive positive pressure ventilation (NIPPV) has a very high failure rate in patients with moderate to severe ARDS and is associated with poor outcomes $(18,19)$. In patients with AHRF, HFNC is 


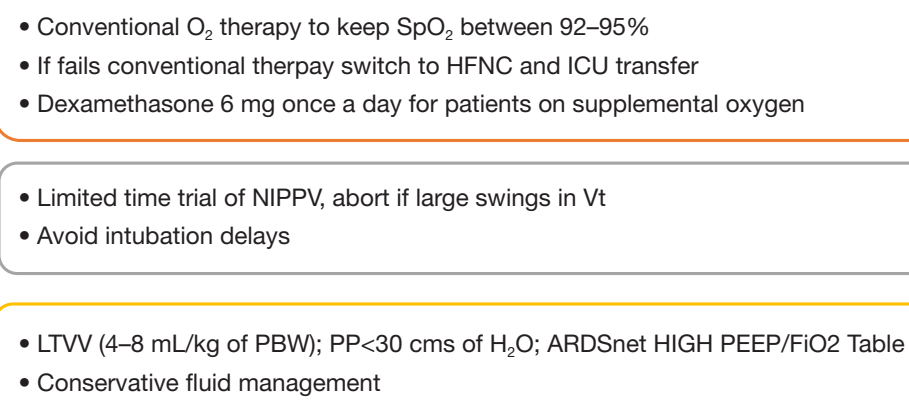

- LTVV (4-8 mL/kg of PBW); PP<30 cms of $\mathrm{H}_{2} \mathrm{O}$; ARDSnet HIGH PEEP/FiO2 Table

- Conservative fluid management

- PPV for atleast 16 hours a day; NMB pushes for ventilator dyssynchrony

- Rescue-RM; ECMO to be reserved for selected cases depending on eligibility and chances for survival - NO inhaled vasodilators

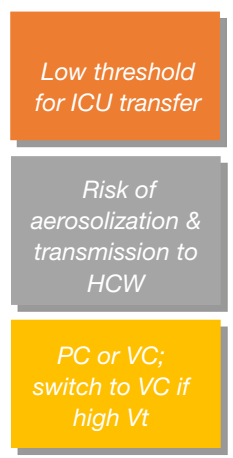

$P F<150$ $\mathrm{FiO}_{2}<0.6 ;$ ARDS onset $<48$ hours

$>1$ hour of stable optimal therapy \& $\mathrm{PP}>30, \mathrm{FiO} 2>0.8$ or $\mathrm{pH}<7.2$

Figure 1 Overview of management of COVID-19 respiratory failure. COVID-19, coronavirus disease 2019.

recommended over NIPPV for achieving target oxygen saturation. Delay in timely initiation of mechanical ventilation is associated with worse outcomes in viral pneumonias. If adequate oxygenation is not achieved by HFNC, intubation should never be delayed for a trial of NIPPV $(20,21)$. A recent study found that in patients with moderate or severe AHRF on NIPPV, the median swing in esophageal pressure was $34 \mathrm{cmH}_{2} \mathrm{O}$ and the median exhaled tidal volume was $11 \mathrm{~mL} / \mathrm{kg}$ of predicted body weight (PBW) (22). Such large swings in transpulmonary pressures, generating unsafe tidal volumes, can further aggravate the lung injury (23). Also, NIPPV is a high aerosol generating procedure associated with nosocomial transmission to healthcare providers [odds ratio (OR) 3.1; 95\% CI, 1.4-6.8] (24), and should be used with caution in the correct clinical (i.e., short term trial) and operational context (surge situations with depleted resources).

\section{Awake prone ventilation with $\mathrm{O} 2$ therapy}

A recently published study prior to the COVID-19 pandemic $(n=20)$ showed that early application of awake prone positioning with HFNC prevented intubation in patients with moderate ARDS (25). Since the pandemic, multiple studies have tried to evaluate the effect of awake proning in AHRF patients with COVID-19. In a study on 50 non intubated patients with COVID-19,
Caputo and colleagues (26) found a statistical increase in $\mathrm{SpO}_{2} 5$ minutes after awake proning [preproning: 84\%; interquartile range (IQR), 75-85\%; postproning: 94\%; IQR, 90-95\%; $\mathrm{P}=0.001]$. Sartini and colleagues showed improvement in respiratory rates $(\mathrm{P}<0.001)$ and $\mathrm{SpO}_{2}$ and $\mathrm{PF}$ ratios $(\mathrm{P}<0.001)$ after awake proning in patients who were hypoxemic despite being on NIPPV (27). In another observational study, awake proning improved oxygenation in only $25 \%$ of the patients (28). Finally, Thomson et al. had similar findings of improvement in $\mathrm{SpO}_{2} 1$ hour after initiation of prone position in spontaneously breathing patients with COVID-19 severe AHRF (29). Although early improvement in physiological parameter like oxygenation looks promising, none of the studies were randomized and did not evaluate patient centered or long term mortality outcomes. Relevant concerns have been raised that the use of awake proning could potentially delay the intubation (30), which has been associated with increased mortality in patients with ARDS (31). The safety of this procedure in diverse populations and the overall rates of eventual intubation are also significant safety end points that have not been adequately studied at this time. So, in the absence of a randomized trial, this evidence is weak at best with insufficient evidence in support its efficacy or safety. Due to the rapid fulminant progression of this disease, intubation should not be delayed for a trial of such 
unproven therapies.

\section{Management of ARDS}

\section{COVID-19 ARDS: an impostor?}

Gattinoni et al. have recently hypothesized that COVID-19 pneumonia has two distinct dynamic phases with very different physiologic mechanisms and thus different responses to management strategies $(32,33)$. The initial phase is described as type L COVID-19 pneumonia which is characterized by low elastance or normal compliance, low ventilation to perfusion ratio, low lung weight and low lung recruitability. There have been suggestions to treat the early phase with low PEEP and less restrictive tidal volume strategy. However, these are preliminary reports with minimal supporting data confirming such assertions. Based on the evidence we have, mechanically ventilated patients should continue to be managed with low tidal volumes (LTV) and high PEEP strategies as the initial intervention. If $\mathrm{L}$ type is suspected, PEEP could be individualized for each patient with careful monitoring of plateau pressure, driving pressure and compliance of the respiratory system (34).

With continued inflammation, the alveolar capillary membrane permeability increases leading to increased interstitial edema, increased lung weight and dependent atelectasis. Type H COVID-19 pneumonia or the typical ARDS is characterized by high elastance, high right-to-left shunt, increased lung weight and high recruitability. The two types could be differentiated by obtaining computerized tomography (CT) imaging of the lungs. However, routine use of CT to titrate PEEP is neither practical nor feasible in COVID-19 patients due to logistical (transporting a highly contagious mechanically ventilated patient to the radiology suite and the antecedent risk of exposure) and operational reasons in most health care systems. Instead, surrogate measures of the respiratory system mechanics (i.e., plateau pressure, driving pressure and compliance) as calculated on the ventilators should be used to set inflation pressures while being mindful of the twin dictates of ventilator management in ARDS, namely limiting volutrauma and barotrauma.

\section{Mechanical ventilation}

Patients need to be committed to ARDSnet LTV lung protective ventilation strategies, upon initiation of mechanical ventilation. LTV reduces relative risk of death by as much as $30 \%$ (35), if maintained within a range of
4-8 $\mathrm{mL} / \mathrm{kg}$ of $\mathrm{PBW}(15)$. The societal guidelines recommend tidal volumes at $6-8 \mathrm{~mL} / \mathrm{kg}$. Higher volumes $(8 \mathrm{~mL} / \mathrm{kg}$ ) are permitted with ventilator asynchrony (double triggering) or if there is evidence of flow starvation (35). Plateau pressures $<30 \mathrm{cms}$ of $\mathrm{H} 2 \mathrm{O}$ should be targeted.

The predominant feature of COVID-19 associated ARDS is a dramatic response to a high PEEP strategy, with recruitment of the lung parenchyma. High PEEP strategies have shown improvements in oxygenation in ARDS patients and its use in moderate to severe ARDS may be associated with a lower mortality $(36,37)$. The use of a modified high PEEP ARDSnet PEEP: FiO2 table (38) should be considered for these patients, if clinically relevant. These patients should be placed on high PEEP, and FiO2 should be weaned quickly. Driving pressure (plateau pressure PEEP) monitoring has also been proposed as a target for safe ventilation (39). To date, there are no prospective studies showing better outcomes when using driving pressure as a targeting variable for management of ARDS patients.

\section{Fluid management}

Prior data from ARDS trials have clearly demonstrated better outcomes associated with conservative fluid management (40). Absence of a clear mortality benefit does not offset the benefit accrued in terms of increased ventilator free days $(14.6 \pm 0.5$ vs. $12.1 \pm 0.5$ days, $\mathrm{P}<0.001)$ and decreased ICU length of stay in patients $(13.4 \pm 0.4 v s$. $11.2 \pm 0.4$ days, $\mathrm{P}<0.001)$. In the absence of clinical shock, attempting a net negative fluid balance using FACTT Lite protocol (41) is recommended for these patients.

\section{Prone position}

Due to the high potential for recruitment of the lung parenchyma, early prone position (PP) should be considered for patients with moderate to severe ARDS $\left(\mathrm{PaO}_{2}: \mathrm{FiO}_{2}\right.$ $<150$, on $\mathrm{FiO}_{2} \geq 0.6$ ) (15). In a landmark trial (42), early prone position ventilation showed a reduction in 28-day mortality by $17 \%$ and a subsequent meta-analysis (43) confirmed these findings. PPV should be implemented early (within 48 hours of ARDS onset) in the disease course when the lung injury is still reversible. PPV leads to overall improvement in lung recruitment as the increased aeration and recruitment of the dorsal regions tend to exceed the decreased aeration and derecruitment of the ventral regions. This leads to improved oxygenation and $\mathrm{CO}_{2}$ clearance. PPV also modulates ventilator induced lung injury (VILI) by redistribution and homogenization of 'strain' across 
the lung tissue $(44,45)$. Proning results in a favorable redistribution of transpulmonary pressures which helps establish and sustain recruitment in response to PEEP. PP also has a favorable effect on hemodynamics by reversing acute cor-pulmonale (46) and increasing the venous return (47). $\mathrm{PP}$ is a safe procedure which can be easily applied in most patients without any significant risks. However, these patients require significant care to prevent pressure ulcers, compression of nerves and retinal vessels and corneal abrasions. Catastrophic events such as endotracheal tube and other catheter dislodgements, also need to be considered/monitored during these sessions. Nevertheless, these complications can be easily prevented by a vigilant nursing and 'respiratory therapist' driven protocols aimed at frequent assessments and repositioning of the body areas under stress (48). Institutions should use manual PP for all patients as the use of automated beds has never been studied in clinical settings, and in pandemic situations manual proning would be the most effective way for resource utilization in these patients.

\section{Other adjunctive/rescue interventions}

\section{Neuromuscular blocking agents (NMBA)}

NMBA like cisatracurium were previously employed as continuous infusions for management of patients with ARDS (49). However, current literature (50) has questioned that use with no difference in 90 -day mortality $(42.5 \%$ vs. $42.8 \%, 95 \% \mathrm{CI},-6.4$ to $5.9 ; \mathrm{P}=0.93)$. We advocate the use of NMBAs, if ventilator dyssynchrony persists despite optimal use of analgesia and sedation. NMBA boluses like rocuronium, with deliberate attempts at ventilator manipulation to offset the dyssycnhrony should be considered as the initial treatment of choice as opposed to a continuous infusion (15).

\section{Recruitment maneuvers}

Various guidelines have issued conditional recommendations for use of recruitment maneuvers (RM) $(15,35)$. If RM are used, current evidence suggests use of routine recruitment over step wise incremental RM. RM are fraught with complications (i.e., barotrauma and hemodynamic collapse), and as such avoided in patients in 'shock' or those with a tenuous hemodynamic profile. There is no data to support the routine use of inhaled nitric oxide or pulmonary vasodilators in ARDS management.

\section{Extra corporeal membrane oxygenation (ECMO)}

Role of ECMO is not well defined in the paradigm of ARDS management. A recent Bayesian analysis (51) of a prior trial (52) and a meta-analysis (53) have shown improved mortality (meta-analysis $34 \%$ vs. $47 \%$; RR $0.73,95 \% \mathrm{CI}, 0.58-0.92 ; \mathrm{P}=0.008)$ in patients with severe ARDS. ECMO is a resource intensive intervention available only in select hospitals. Expert centers should adopt an evidence based ARDS protocol algorithm in concert with a multi-disciplinary team approach, upon failure of conventional therapy to decide on ECMO eligibility. ECMO should be offered to only those with the highest chance of survival.

\section{Corticosteroids}

A large randomized control trial conducted by RECOVERY Collaborative Group showed that the patients infected with SARS-CoV-2, who received dexamethasone had a significantly lower 28 -day mortality $(21.6 \%$ vs. $24.6 \%$; $\mathrm{P}<0.001)$, the effect being more pronounced in patients receiving invasive mechanical ventilation $(29.0 \%$ vs. $40.7 \%$; $\mathrm{P}<0.001)$ and supplemental oxygen $(21.5 \%$ vs. $25.0 \%$; $\mathrm{P}=0.022$ ) (54). Based on this overwhelmingly positive evidence, we suggest the routine use of dexamethasone in patients with COVID-19 who present with respiratory failure requiring supplemental oxygen or mechanical ventilation.

\section{Care of mechanically ventilated patients}

High efficiency particulate air (HEPA) filters are mechanical type filters that collect particles through several different mechanisms, including impaction, interception, gravity and diffusion. Particles collected on the filter are trapped in the filter matrix and are not easily released from the upstream (outward) side of the filter. These filters are tested for particles of sizes up to 0.2 to 0.3 microns. They can trap the droplets that are released during the aerosolizing procedures like bag mask ventilation and mechanical ventilation (55). Therefore, HEPA filters should be attached to the BMV assembly and ventilator circuit to prevent the dispersion of the droplets. Unnecessary disconnection of the ventilator from a patient with COVID-19 should be minimized to avoid derecruitment and needless exposure of virus to the environment. Mechanical ventilation and other care for patients with acute respiratory failure are associated with 
a higher risk of nosocomial transmission so healthcare providers need to adhere with appropriate personal protective equipment (PPE) as they take care of these patients. Ventilator circuits need to have tight seals to prevent higher risk of aerosolization. Ventilator monitors can be placed outside the room, to allow for frequent ventilator adjustments while simultaneously decreasing the risk of exposure to staff. Other general supportive care related to sedation practices, delirium prevention and infection surveillance should be based on standard ICU practice and protocols.

\section{Other causes of respiratory failure}

Like most other viral infections, COVID-19 pneumonia can lead to both COPD and asthma exacerbations (56). SARS$\mathrm{CoV}-2$ infections can trigger an inflammatory reaction triggering COPD and asthma exacerbations and should be managed with corticosteroids and bronchodilators, based on best practice guidelines for the underlying disease processes.

Initial observational data has suggested abnormal coagulation patterns in COVID-19 patents. Studies show that $30-43 \%$ of these patients develop clinically relevant thrombotic complications despite the use of standard venous thromboembolic (VTE) prophylaxis $(57,58)$. COVID-19 patients who otherwise have minimal lung involvement could potentially present with dyspnea and hypoxemia which may be triggered by pulmonary embolism and right heart failure. Coagulation profile patients with COVID-19 should be monitored closely. Elevated D-Dimers at baseline or an increase during hospitalization have been associated with increased mortality. Some authors have recommended escalated doses of VTE prophylaxis for patients with elevated or increasing D-Dimer levels (34).

Increased mortality with COVID-19 cardiac complications of SARS-CoV-2 infection include severe arrhythmias, heart failure exacerbations and myocarditis as a result of direct myocardial injury leading to cardiogenic shock. These patients can present with signs and symptoms ranging from mild dyspnea, acute pulmonary edema to sudden cardiac death. The mechanisms underlying myocardial injury remain unknown and could reflect a systemic inflammatory process. Patients with suspected myocardial injury or myocarditis should get a 12-lead EKG on initial visit and as needed, serum high-sensitivity troponin, NT-proBNP, and echocardiography to assess for global and regional wall motion abnormalities and function (59).

\section{Conclusions}

In summary evidence-based treatment strategies that are the bedrock of COVID-19 ARDS management cannot be deferred, for unproven and untested therapies; except as part of a scientific trial or clinical experiment. Treatment of COVID-19 induced ARDS should rely on established best practices and guidelines. At present there are no specific therapies proven to be of benefit other than supportive care based on $\mathrm{O}_{2}$ supplementation and mechanical ventilation. Interventional trials in critical care are frequently plagued with negative results (60), a sobering thought, that cannot be discounted. Physicians attuned to scientific pursuit of investigating newer modalities and therapeutic interventions in a desperate attempt to mitigate the death toll associated with the pandemic, should not throw caution to the wind. Similarly, the age-old adage "As to diseases, make a habit of two things-to help, or at least to do no harm-primum non nocere (61)" still holds true. Endorsement and adoption of well-intentioned therapies bereft of evidence does not bode well for our patients and could bring ill repute to the practice of medicine, a damage, if perpetrated, will be tough to rectify and echo through time.

\section{Acknowledgments}

Funding: None.

\section{Footnote}

Reporting Checklist: The authors have completed the Narrative Review reporting checklist. Available at http:// dx.doi.org/10.21037/atm-20-4633

Peer Review File: Available at http://dx.doi.org/10.21037/ atm-20-4633

Conflicts of Interest: All authors have completed the ICMJE uniform disclosure form (available at http://dx.doi. org/10.21037/atm-20-4633). The authors have no conflicts of interest to declare.

Etbical Statement: The authors are accountable for all aspects of the work in ensuring that questions related to the accuracy or integrity of any part of the work are appropriately investigated and resolved. 
Open Access Statement: This is an Open Access article distributed in accordance with the Creative Commons Attribution-NonCommercial-NoDerivs 4.0 International License (CC BY-NC-ND 4.0), which permits the noncommercial replication and distribution of the article with the strict proviso that no changes or edits are made and the original work is properly cited (including links to both the formal publication through the relevant DOI and the license). See: https://creativecommons.org/licenses/by-nc-nd/4.0/.

\section{References}

1. Gorbalenya AE, Baker SC, Baric RS, et al. The species Severe acute respiratory syndrome-related coronavirus: classifying 2019-nCoV and naming it SARS-CoV-2. Nat Microbiol 2020;5:536-44.

2. WHO Director-General's opening remarks at the media briefing on COVID-19: 11 March 2020. Published March 11, 2020. Accessed March 30, 2020. [Internet]. Available online: https://www.who.int/dg/speeches/detail/ who-director-general-s-opening-remarks-at-the-mediabriefing-on-covid-19-11-march-2020

3. COVID-19 Dashboard by the Center for Systems Science and Engineering (CSSE) at Johns Hopkins University (JHU) [Internet]. Available online: https://coronavirus.jhu. edu/map.html

4. Coronavirus Pandemic (COVID-19) [Internet]. OurWorldInData.org 2020. Available online: https:// ourworldindata.org/coronavirus

5. Wu Z, McGoogan JM. Characteristics of and Important Lessons From the Coronavirus Disease 2019 (COVID-19) Outbreak in China: Summary of a Report of 72314 Cases From the Chinese Center for Disease Control and Prevention. JAMA 2020;323:1239-42.

6. Richardson S, Hirsch JS, Narasimhan M, et al. Presenting Characteristics, Comorbidities, and Outcomes Among 5700 Patients Hospitalized With COVID-19 in the New York City Area. JAMA 2020;323:2052-9.

7. Grasselli G, Zangrillo A, Zanella A, et al. Baseline Characteristics and Outcomes of 1591 Patients Infected With SARS-CoV-2 Admitted to ICUs of the Lombardy Region, Italy. JAMA 2020;323:1574-81.

8. Duggal A, Pinto R, Rubenfeld G, et al. Global Variability in Reported Mortality for Critical Illness during the 200910 Influenza A(H1N1) Pandemic: A Systematic Review and Meta-Regression to Guide Reporting of Outcomes during Disease Outbreaks. PLoS One 2016;11:e0155044.

9. Robert Langerth. No Title. Bloomberg [Internet] 2020
Apr 22; Available online: https://www.bloomberg.com/ news/articles/2020-04-22/almost-9-in-10-covid-19patients-on-ventilators-died-in-study

10. Mason RJ. Pathogenesis of COVID-19 from a cell biology perspective. Eur Respir J 2020;5 5:2000607.

11. Sarzani R, Giulietti F, Di Pentima C, et al. Disequilibrium between the classic renin-angiotensin system and its opposing arm in SARS-CoV-2-related lung injury. Am J Physiol Lung Cell Mol Physiol 2020;319:L325-36.

12. Zhu N, Zhang D, Wang W, et al. A novel coronavirus from patients with pneumonia in China, 2019. N Engl J Med 2020;382:727-33.

13. Cavallo JJ, Donoho DA, Forman HP, et al. Hospital Capacity and Operations in the Coronavirus Disease 2019 (COVID-19) Pandemic-Planning for the Nth Patient. JAMA Heal Forum 2020;1:e200345.

14. Rice TW, Janz DR. In Defense of Evidence-Based Medicine for the Treatment of COVID-19 ARDS. Ann Am Thorac Soc 2020;17:787-9.

15. Alhazzani W, Møller MH, Arabi YM, et al. Surviving Sepsis Campaign: Guidelines on the Management of Critically Ill Adults with Coronavirus Disease 2019 (COVID-19). Crit Care Med 2020;48:e440-69.

16. Hick JL, Einav S, Hanfling D, et al. Surge Capacity Principles: Care of the Critically Ill and Injured During Pandemics and Disasters: CHEST Consensus Statement. Chest 2014;146:e1S-e16S.

17. Rochwerg B, Granton D, Wang DX, et al. High flow nasal cannula compared with conventional oxygen therapy for acute hypoxemic respiratory failure: a systematic review and meta-analysis. Intensive Care Med 2019;45:563-72.

18. Bellani G, Laffey JG, Pham T, et al. Noninvasive Ventilation of Patients with Acute Respiratory Distress Syndrome. Insights from the LUNG SAFE Study. Am J Respir Crit Care Med 2017;195:67-77.

19. Mosier JM, Sakles JC, Whitmore SP, et al. Failed noninvasive positive-pressure ventilation is associated with an increased risk of intubation-related complications. Ann Intensive Care 2015;5:4.

20. Alraddadi BM, Qushmaq I, Al-Hameed FM, et al. Noninvasive ventilation in critically ill patients with the Middle East respiratory syndrome. Influenza Other Respir Viruses 2019;13:382-90.

21. Arabi YM, Arifi AA, Balkhy HH, et al. Clinical Course and Outcomes of Critically Ill Patients With Middle East Respiratory Syndrome Coronavirus Infection. Ann Intern Med 2014;160:389-97.

22. Tonelli R, Fantini R, Tabbì L, et al. Inspiratory Effort 
Assessment by Esophageal Manometry Early Predicts Noninvasive Ventilation Outcome in de novo Respiratory Failure: A Pilot Study. Am J Respir Crit Care Med 2020;202:558-67.

23. Slutsky AS, Ranieri VM. Ventilator-Induced Lung Injury. N Engl J Med 2013;369:2126-36.

24. Tran K, Cimon K, Severn M, et al. Aerosol generating procedures and risk of transmission of acute respiratory infections to healthcare workers: a systematic review. PLoS One 2012;7:e35797.

25. Ding L, Wang L, Ma W, et al. Efficacy and safety of early prone positioning combined with HFNC or NIV in moderate to severe ARDS: a multi-center prospective cohort study. Crit Care2020;24:28.

26. Caputo ND, Strayer RJ, Levitan R. Early Self-Proning in Awake, Non-intubated Patients in the Emergency Department: A Single ED's Experience During the COVID-19 Pandemic. Acad Emerg Med 2020;27:375-8.

27. Sartini C, Tresoldi M, Scarpellini P, et al. Respiratory Parameters in Patients With COVID-19 After Using Noninvasive Ventilation in the Prone Position Outside the Intensive Care Unit. JAMA 2020;323:2338-40.

28. Elharrar X, Trigui Y, Dols AM, et al. Use of Prone Positioning in Nonintubated Patients With COVID-19 and Hypoxemic Acute Respiratory Failure. JAMA 2020;323:2336-8.

29. Thompson AE, Ranard BL, Wei Y, et al. Prone Positioning in Awake, Nonintubated Patients With COVID-19 Hypoxemic Respiratory Failure. JAMA Intern Med 2020;e203030.

30. Sarma A, Calfee CS. Prone Positioning in Awake, Nonintubated Patients With COVID-19: Necessity Is the Mother of Invention. JAMA Intern Med 2020. [Epub ahead of print].

31. Kangelaris KN, Ware LB, Wang CY, et al. Timing of Intubation and Clinical Outcomes in Adults With Acute Respiratory Distress Syndrome. Crit Care Med 2016;44:120-9.

32. Gattinoni L, Coppola S, Cressoni M, et al. Covid-19 Does Not Lead to a "Typical" Acute Respiratory Distress Syndrome. Am J Respir Crit Care Med 2020;201:1299-300.

33. Gattinoni L, Chiumello D, Caironi P, et al. COVID-19 pneumonia: different respiratory treatment for different phenotypes? Intensive Care Med 2020;46:1099-102.

34. Kallet RH. Should PEEP Titration Be Based on Chest Mechanics in Patients With ARDS? Respir Care 2016;61:876-90.
35. Fan E, Del Sorbo L, Goligher EC, et al. An Official American Thoracic Society/European Society of Intensive Care Medicine/Society of Critical Care Medicine Clinical Practice Guideline: Mechanical Ventilation in Adult Patients with Acute Respiratory Distress Syndrome. Am J Respir Crit Care Med 2017;195:1253-63.

36. Walkey AJ, Del Sorbo L, Hodgson CL, et al. Higher PEEP versus Lower PEEP Strategies for Patients with Acute Respiratory Distress Syndrome. A Systematic Review and Meta-Analysis. Ann Am Thorac Soc 2017;14:S297-303.

37. Briel M, Meade M, Mercat A, et al. Higher vs. Lower Positive End-Expiratory Pressure in Patients With Acute Lung Injury and Acute Respiratory Distress Syndrome: Systematic Review and Meta-analysis. JAMA 2010;303:865-73.

38. NIH NHLBI ARDS Clinical Network Mechanical Ventilation Protocol Summary [Internet]. Available online: http://www.ardsnet.org/files/ventilator_ protocol_2008-07.pdf

39. Amato MBP, Meade MO, Slutsky AS, et al. Driving Pressure and Survival in the Acute Respiratory Distress Syndrome. N Engl J Med 2015;372:747-55.

40. National Heart, Lung, and Blood Institute Acute Respiratory Distress Syndrome (ARDS) Clinical Trials Network, Wiedemann HP, Wheeler AP, et al. Comparison of Two Fluid-Management Strategies in Acute Lung Injury. N Engl J Med 2006;354:2564-75.

41. Grissom CK, Hirshberg EL, Dickerson JB, et al. Fluid management with a simplified conservative protocol for the acute respiratory distress syndrome. Crit Care Med 2015;43:288-95.

42. Guérin C, Reignier J, Richard JC, et al. Prone Positioning in Severe Acute Respiratory Distress Syndrome. N Engl J Med 2013;368:2159-68.

43. Munshi L, Del Sorbo L, Adhikari NKJ, et al. Prone Position for Acute Respiratory Distress Syndrome. A Systematic Review and Meta-Analysis. Ann Am Thorac Soc 2017;14:S280-8.

44. Guérin C. Prone positioning acute respiratory distress syndrome patients. Ann Transl Med 2017;5:289.

45. Gattinoni L, Taccone P, Carlesso E, et al. Prone Position in Acute Respiratory Distress Syndrome. Rationale, Indications, and Limits. Am J Respir Crit Care Med 2013;188:1286-93.

46. Vieillard-Baron A, Charron C, Caille V, et al. Prone Positioning Unloads the Right Ventricle in Severe ARDS. Chest 2007;132:1440-6. 
47. Jozwiak M, Teboul JL, Anguel N, et al. Beneficial Hemodynamic Effects of Prone Positioning in Patients with Acute Respiratory Distress Syndrome. Am J Respir Crit Care Med 2013;188:1428-33.

48. Oliveira VM de, Weschenfelder ME, Deponti G, et al. Good practices for prone positioning at the bedside: Construction of a care protocol. Rev Assoc Med Bras (1992) 2016;62:287-93.

49. Papazian L, Forel JM, Gacouin A, et al. Neuromuscular Blockers in Early Acute Respiratory Distress Syndrome. N Engl J Med 2010;363:1107-16.

50. National Heart, Lung, and Blood Institute PETAL Clinical Trials Network, Moss M, Huang DT, et al. Early Neuromuscular Blockade in the Acute Respiratory Distress Syndrome. N Engl J Med 2019;380:1997-2008.

51. Goligher EC, Tomlinson G, Hajage D, et al. Extracorporeal Membrane Oxygenation for Severe Acute Respiratory Distress Syndrome and Posterior Probability of Mortality Benefit in a Post Hoc Bayesian Analysis of a Randomized Clinical Trial. JAMA 2018;320:2251-9.

52. Combes A, Hajage D, Capellier G, et al. Extracorporeal Membrane Oxygenation for Severe Acute Respiratory Distress Syndrome. N Engl J Med 2018;378:1965-75.

53. Munshi L, Walkey A, Goligher E, et al. Venovenous extracorporeal membrane oxygenation for acute respiratory distress syndrome: a systematic review and meta-analysis. Lancet Respir Med 2019;7:163-72.

54. Horby P, Lim WS, Emberson J, et al. Effect of

Cite this article as: Vashisht $\mathrm{R}$, Krishnan S, Duggal A. A narrative review of non-pharmacological management of SARSCoV-2 respiratory failure: a call for an evidence based approach. Ann Transl Med 2020;8(23):1599. doi: 10.21037/atm-20-4633
Dexamethasone in Hospitalized Patients with COVID-19: Preliminary Report. N Engl J Med 2020;NEJMoa2021436.

55. HEPA Filters for COVID-19 [Internet]. Available online: https://www.bullard.com/respiratory/hepa-filters-forcovid-19

56. Hewitt R, Farne H, Ritchie A, et al. The role of viral infections in exacerbations of chronic obstructive pulmonary disease and asthma. Ther Adv Respir Dis 2016;10:158-74.

57. Klok FA, Kruip MJHA, Meer NJM Van Der, et al. Incidence of thrombotic complications in critically ill ICU patients with COVID-19. Thromb Res 2020;191:145-7.

58. Helms J, Tacquard C, Severac F, et al. Intensive Care Medicine High risk of thrombosis in patients in severe SARS-CoV-2 infection: a multicenter prospective cohort study High risk of thrombosis in patients in severe SARSCoV-2 infection: a multicenter prospective cohort study. Intensive Care Med 2020;46:1089-98.

59. Guzik TJ, Mohiddin SA, Dimarco A, et al. COVID-19 and the cardiovascular system: implications for risk assessment, diagnosis, and treatment options. Cardiovasc Res 2020;116:1666-87.

60. Santacruz CA, Pereira AJ, Celis E, et al. Which Multicenter Randomized Controlled Trials in Critical Care Medicine Have Shown Reduced Mortality? A Systematic Review. Crit Care Med 2019;47:1680-91.

61. Gifford RW Jr. Primum Non Nocere. JAMA 1977;238:589-90. 